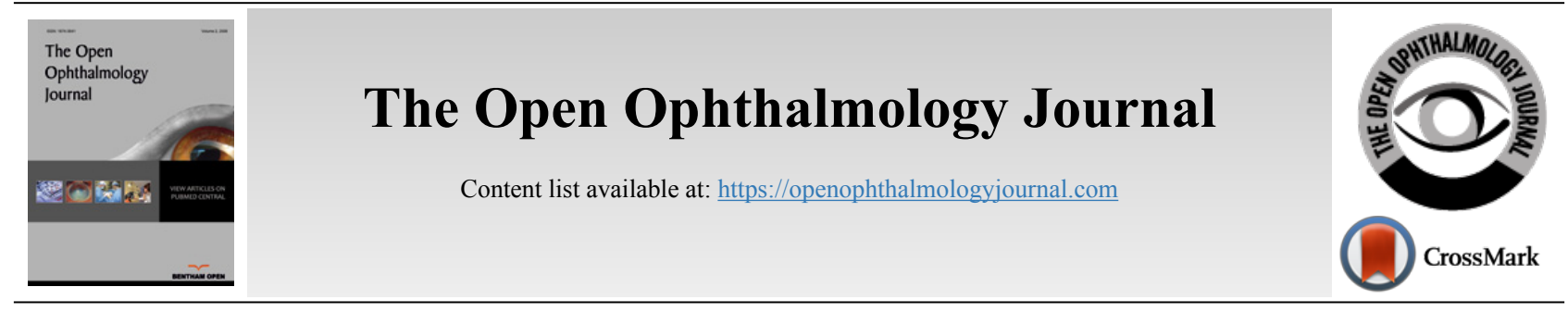

RESEARCH ARTICLE

\title{
Prophylactic Circumferential Laser Retinopexy in Macular Hole and Epiretinal Membrane Surgeries
}

Oded Ohana $^{1, * \#}$, Ran Rutenberg ${ }^{1, \#}$, Elad Moisseiev ${ }^{2}$, Zvi Davidovich $^{3}$, Anat Lowenstein ${ }^{1}$, Shulamit Schwartz ${ }^{1}$ and Adiel Barak $^{1}$

${ }^{1}$ Department of Ophthalmology, Tel Aviv Sourasky Medical Center, Sackler Faculty of Medicine, Tel Aviv University, Tel Aviv, Israel

${ }^{2}$ Department of Ophthalmology, Meir Medical Center, Kfar-Saba, Israel

${ }^{3}$ Schneider Children's Medical Center in Israel, Petach-Tikvah, Israel

\begin{abstract}
:
Background:

The role of PCLR in vitrectomy surgeries for macular diseases is controversial. Several studies report a reduction in post-operative RD rates, while other studies do not show such reduction. reducing complications stemming from intra- and post- operative retinal tears, especially at sclerotomy sites, are suggested as reasons for PCLR use.

Objective:

To investigate the effect of PCLR on the rate of post-operative RD in patients undergoing pars plana vitrectomy for either Macular Hole (MH) or epiretinal membrane (ERM).

Methods:

This retrospective study included 179 eyes of 170 patients who underwent vitrectomy for either MH or ERM between 2006-2012. Recorded parameters included demographic information, diagnosis, lens status, use and type of tamponade, whether PCLR was performed, follow-up period, the occurrence of RD, the cause and time of RD. Outcome and complication rates were compared between patients who received PCLR (PCLR group) and those who did not (Control group).

Results:

The study included 179 eyes of 170 patients. 126 eyes had PCLR while 53 eyes did not. Pre-operative demographics data, best-corrected visual acuity, lens status and indication for macular surgery were similar between the two groups. Post-operative RD was diagnosed in one eye (1.8\%) in the control group and 5 eyes $(3.9 \%)$ in the PCLR group (Kaplan-Meier survival analysis, log-rank $p=0.28$ ).

Conclusion:

PCLR was not correlated with a decrease in RD in MH and ERM surgeries in our cohort. Intra-operative and post-operative examination for small retinal breaks is essential in all surgeries. PCLR might be unnecessary in small gauge vitrectomies.
\end{abstract}

Keywords: Circumferential laser barrage, Epiretinal membrane, Macular hole, Pars plana vitrectomy, Retinal detachment, Retinopexy, Prophylactic.

Article History

Received: August 25, 2019 Revised: January 14, 2020

Accepted: January 24,2020

\section{INTRODUCTION}

Retinal Detachment (RD) is a possible severe complication of macular surgeries. Previous studies report the incidence of

* Address correspondence to this author at the Department of Ophthalmology, Tel Aviv Medical Center, 6 Weizmann St., Tel-Aviv 64236, Israel.

Tel: +972-3-6973408, Fax: +972-3-6973870, E-mail: odedoh@hotmail.com

${ }^{*}$ Equal contribution
RD following Macular Hole (MH) repair to be $6.6 \%-11 \%$ [1, $2]$, and the incidence of $\mathrm{RD}$ following epiretinal membrane (ERM) surgeries to be $2.5 \%-6 \%[1,3]$. However, recent data suggest that the incidence of RD with modern surgical equipment and technique may be as low as $2.4 \%$ and $1.0 \%$ for MH and ERM, respectively [4, 5].

Different methods have been proposed to prevent RD after Pars-Plana-Vitrectomy (PPV) surgeries, for example, careful 
peeling of posterior hyaloid and meticulous intraoperative detection and treatment of retinal breaks [6]. The literature also includes relatively drastic measures, such as prophylactic scleral buckling [7].

In 2007, Koh et al. first used intraoperative prophylactic circumferential laser retinopexy (PCLR) and showed a threefold reduction in the incidence of post-operative RD [8]. Further studies report conflicting results for PCLR use. While several reports show a beneficial effect for PCLR [9, 10], other reports fail to show a similar effect [11].

We sought to evaluate the efficacy of PCLR for the prevention of RD in patients undergoing PPV for macular diseases, based on the experience gained in our department.

\section{MATERIALS AND METHODS}

We conducted a retrospective, comparative study. The study adhered to the tenets of the declaration of Helsinki and was approved by the institutional review board of Tel Aviv Medical Center, Tel Aviv, Israel. We reviewed the medical records of patients who had PPV for MH or ERM surgeries from January 2006 to December 2012. Exclusion criteria were any history of severe ocular disease, high myopia (spherical equivalents of more than $-6 \mathrm{D}$ or an axial length greater than $26.5 \mathrm{~mm}$ ), uveitis, posterior capsular tear, and any kind of intraocular silicone oil use. Bilateral cases were both included in the analysis, as we considered these to be unrelated events.

The cohort was divided into two groups: the PCLR group consisted of patients who had PCLR during PPV for MH or ERM, and the control group consisted of patients who did not receive PCLR. At our department, starting from January 2008, following the publication of Koh et al. [8], PCLR was used in the majority of macular surgeries. One surgeon (A.B.) routinely performed PCLR for MH and ERM, whereas other surgeons did not use PCLR routinely. The control group was mainly composed of surgeries performed from January 2006 to January 2008.

Data extracted from patients files included: pre-operative demographic data, Best-Corrected Visual Acuity (BCVA), lens status, and diabetes mellitus status. Operative data included whether phacoemulsification was performed, type of tamponade, and the use of PCLR. Follow up data included the occurrence of $\mathrm{RD}$, elevated IOP, hypotony and need for further surgery.

All operations were performed by one of three experienced vitreoretinal surgeons. A standard three port 23 gauge PPV was performed. Initial surgical steps included phacoemulsification and intra ocular lens implantation when cataract removal was indicated, followed by core vitrectomy and induction of posterior vitreous detachment using triamcinolone acetonide (Kenalog, Bristol-Myers Squibb, New-York, USA) when the posterior hyaloid was fully or partially attached to the retina. The Internal Limiting Membrane (ILM) or ERM (for MH and for ERM peeling surgeries, respectively) was stained with membrane dual blue dye $(0.125 \mathrm{mg}$ Brilliant Blue $\mathrm{G}+0.75$ Trypan Blue, Dutch Ophthalmic USA, Exeter, NH, USA). The membrane was peeled using intraocular ILM forceps. In eyes undergoing PCLR, 2-3 rows of moderate intensity laser burns were applied, approximately half a burn width apart, posterior to the vitreous base. At the end of the surgery, a comprehensive examination of the retinal periphery was performed, using scleral indentation when necessary, and any tears noted were treated with laser retinopexy. For MH surgeries, air-fluid exchange was performed and then exchanged to either $16 \%$ $\mathrm{C}_{3} \mathrm{~F}_{8}$ or $20 \% \mathrm{SF}_{6}$ gas. For ERM surgeries, the vitreous cavity was filled with Balanced Salt Solution (BSS). Sclerotomies were not routinely sutured, and according to surgeon's discretion were sutured with 7-0 polyglactin 910 (Coated Vicryl, Ethicon, USA).

The primary outcome was the occurrence of post-operative RD. Secondary outcomes included the incidence of elevated intraocular pressure (IOP), hypotony and endophthalmitis.

Statistical analysis was performed using R Project for Statistical Computing 3.2.3 (R Foundation for Statistical Computing, Vienna, Austria). All comparisons were two-sided. A p-value $<0.05$ was considered significant. Numerical variables were compared using Student's t-test. Categorical variables were compared using wither Fisher's exact test or chi-squared test, as appropriate. Time to retinal detachment was analyzed using Kaplan-Meier log-rank method.

\section{RESULTS}

A total of 179 eyes (170 patients) were analyzed. 126 eyes had PCLR and 53 eyes did not and composed the control group.

In the PCLR group, the mean age was $69.6 \pm 9.0$. Mean follow-up was $23.7 \pm 22.6$ months (median 14.4). Table 1 describes the demographic information and operative characteristics of the two groups. No significant differences were found between groups.

Table 2 presents follow up information for our cohort. No difference was found regarding the proportion of patients that had short follow-up periods.

Table 1. Demographics and Operative Characteristics

\begin{tabular}{|c|c|c|c|}
\hline- & Control Group & PCLR Group & P-Value \\
\hline Eyes & 53 & 126 & - \\
\hline Age (Years) & $70.3 \pm 8.9$ & $70.8 \pm 6.6$ & 0.70 \\
\hline Sex (F) & $25(49 \%)$ & $58(48 \%)$ & 1 \\
\hline Diagnosis & - & - & 0.32 \\
\hline Macular Hole & $19(35.8 \%)$ & $56(44.4 \%)$ & - \\
\hline Epiretinal Membrane & $34(64.1 \%)$ & $70(55.5 \%)$ & - \\
\hline Side (Right) & $26(50 \%)$ & $62(52 \%)$ & 1 \\
\hline
\end{tabular}


(Table 1) contd.....

\begin{tabular}{|c|c|c|}
\hline (Table 1) contd..... & Control Group & PCLR Group \\
\hline Lens Status & - & - \\
\hline Phakic & 37 & 90 \\
\hline Pseudophakic & 15 & 36 \\
\hline Aphakic & 1 & 0 \\
\hline BCVA, LogMAR & - & $0.82 \pm 0.32$ \\
\hline (Snellen Equivalent) & $(20 / 132)$ \\
\hline Diabetes Mellitus & $0.87 \pm 0.33$ & $44(35 \%)$ \\
\hline Phacovitrectomy & $12(23 \%)$ & 0.33 \\
\hline Tamponade & $14(27 \%)$ & $50(40 \%)$ \\
\hline Balanced Saline Solution & - & 0.12 \\
\hline Air & $33(62.2 \%)$ & 0.12 \\
\hline Gas & $2(3.7 \%)$ & 0.24 \\
\hline
\end{tabular}

Table legend: BCVA Best Corrected Visual Acuity; ERM Epiretinal Membrane; MH Macular Hole; PCLR Prophylactic Circumferential Laser Retinopexy

Table 2. Follow-up information

\begin{tabular}{|c|c|c|}
\hline- & Control Group & PClvalue \\
\hline Mean Follow-up (months) & $24.51 \pm 25.03$ & $22.97 \pm 20.32$ \\
\hline Follow up $<2$ months & $2 / 51$ & $5 / 119$ \\
\hline Follow up $<3$ months & $8 / 51$ & $18 / 119$ \\
\hline Follow up $<6$ months & $12 / 51$ & 0.82 \\
\hline Follow up $<12$ months & $22 / 51$ & 1 \\
\hline Follow up $>12$ months & $7 / 51$ & $53 / 119$ \\
\hline
\end{tabular}

Table caption: PCLR prophylactic circumferential laser retinopexy

Table 3. Retinal Detachment Stratified According to Initial Diagnosis and Lens Status:

\begin{tabular}{|c|c|c|c|c|c|c|}
\hline- & \multicolumn{2}{|c|}{ Control Group } & \multicolumn{2}{|c|}{ PCLR Group } & \multirow{2}{*}{$\frac{\text { Total }}{-}$} & \multirow{2}{*}{$\begin{array}{c}\text { P-value } \\
-\end{array}$} \\
\hline- & Phakic & Pseudophakic & Phakic & Pseudophakic & & \\
\hline Macular Hole & $0 / 13$ & $0 / 5$ & $3 / 47$ & $0 / 11$ & $3 / 76$ & 0.56 \\
\hline Epiretinal Membrane & $1 / 24$ & $0 / 10$ & $2 / 43$ & $0 / 25$ & $3 / 103$ & 1 \\
\hline Total & $1 / 37$ & $0 / 15$ & $5 / 90$ & $0 / 36$ & $6 / 179$ & - \\
\hline
\end{tabular}

Table legend: PCLR prophylactic circumferential laser retinopexy

Table 3 shows the incidence of RD in the different groups. Six cases of RD were noted in the cohort, five in the PCLR group and one in the control group $(4.2 \% v s .1 .9 \%$, Chi-square $=0.48)$. All of the patients who developed RD were phakic at the time of surgery. Four underwent phacoemulsification at the time of original PPV, three in the PCLR group and one in the control group. In the PCLR group, post-operative RD developed in 2 eyes with ERM and in 3 eyes with MH, and the control group patient who had an RD had ERM. Phacovitrectomy was not correlated with a higher risk for RD (4/115 vs. 2/64, PPV only vs. phacovitrectomy respectively, Chi-square $=0.90)$. Likewise, lens status was not associated with an increased risk of post-operative RD $(p=0.28)$. PCLR did not significantly reduce RD occurrence in either phakic ( $1 / 37$ vs $5 / 90$ in the control and PCLR groups respectively, chisquare $\mathrm{p}=0.47)$ or pseudophakic eyes $(0 / 15$ vs $0 / 36$ in the control and PCLR groups respectively).

Mean time to retinal detachment in the PCLR group was $49.6 \pm 26.5$ days (range 22-84), while the only RD case in the control group occurred after 379 days. Fig. (1) shows the Kaplan-Meier survival analysis for post-operative RD. No significant survival difference between the groups was found ( $\log$-rank $\mathrm{p}=0.28)$.

Intra-operative retinal tears were detected in 6/53 (11.3\%) of control eyes and in 8/126 (6.3\%) of eyes that underwent PCLR $(\mathrm{P}=0.36)$. One eye in the PCLR group had a retinal break diagnosed and treated intraoperatively, and later developed RD.

Post-operative IOP elevation was noted in 13/179 (7.2\%) of the eyes, all managed successfully with topical agents. Two cases of hypotony $(1.1 \%)$ were noticed, both related to sclerotomy-associated leak, and resolved with suturing of the leaking sclerotomy site. One case of endophthalmitis was seen in our cohort $(0.55 \%)$; the patient was treated with repeat PPV and intra-vitreal antibiotics.

\section{DISCUSSION}

$\mathrm{RD}$ is a devastating complication of vitrectomy surgery. Different techniques aimed at reducing post-operative $\mathrm{RD}$ were suggested as vitrectomy evolved. While some techniques were shown not to be beneficial in reducing post-operative $\mathrm{RD}$, for 


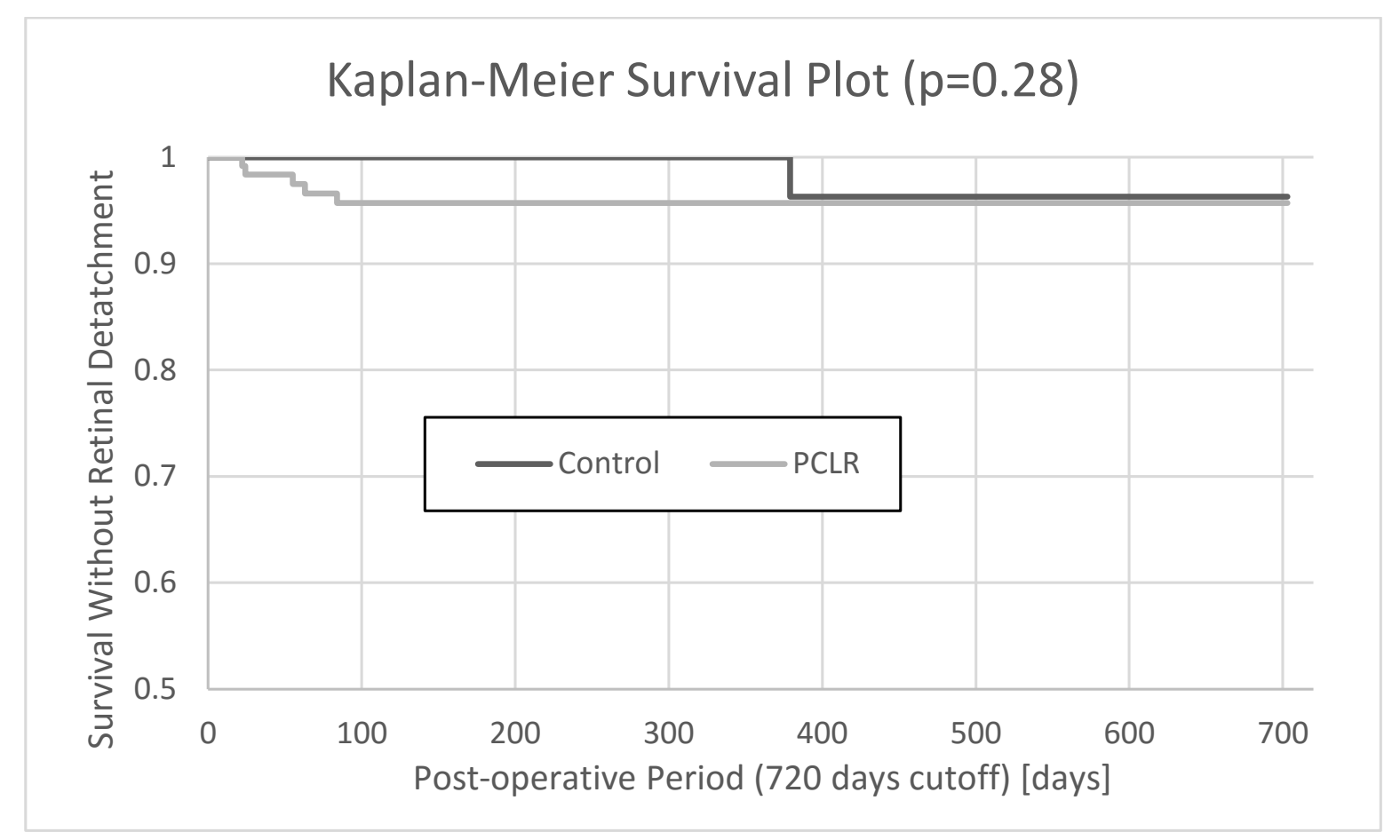

Fig. (1). Kaplan-Meier Survival Analysis. PCLR prophylactic circumferential laser retinopexy.

example Intra-operative sclerotomy-site endolaser retinopexy [11] and pre-operative prophylactic laser retinopexy [12], other techniques did show promise. Koh et al. [8] first suggested PCLR use in 2008, and showed a significant reduction in postoperative RD rate. They hypothesized that applying the laser burns posterior to the sclerotomy sites aided in RD prevention, which could occur if retinal breaks are missed intraoperatively. Further reports by Iwase et al. [10] supported this beneficial effect of PCLR. This study does not show a similar reduction in post-operative RD rates. In fact, although not statistically significant, we saw more post-operative RD cases in the PCLR group (5/126 vs $1 / 53$ in the PCLR and control groups respectively).

It was hypothesized that PCLR would treat any unidentified intraoperative retinal breaks and any potential post-operative break caused by either post-operative vitreal traction, especially at sclerotomy sites $[8,11,13]$, or from the induction of Posterior Vitreous Detachment (PVD) during the surgery [14] Following this rationale, better intra-operative detection and treatment of retinal breaks, or a lower incidence of post-operative retinal breaks will lower the post-operative $\mathrm{RD}$ rates and might obviate the effect of PCLR. This notion is supported by reports showing that deliberate, meticulous intraoperative examination for retinal breaks increases the detection rate of retinal breaks [6], leading to reduced post-operative RD rates. It is possible that Koh et al. [8] and Iwase et al. [10] showed a significant reduction because of a higher baseline RD rate. Compared with these two studies, we had a lower baseline post-operative $\mathrm{RD}$ rate. therefore, finding a statistically significant difference is more challenging.
Technological advances made PPV safer. Recent studies show that 23-gauge vitrectomy surgeries are safer than 20gauge vitrectomy surgeries $[12,15]$. Even more so, less iatrogenic retinal tears are seen in 23-gauge vitrectomy patients. Both Koh et al. [8] and Iwase et al. [10] used a 20gauge vitrectomy system, while we performed 23-gauge surgeries. It is reasonable that the technological improvement in vitrectomy equipment might have made adjunct intraoperative techniques such as PCLR unnecessary.

In our cohort, all post-operative RD occurred in phakic eyes, with three of the cases having phacovitrectomy. While we did not find a statistically significant difference in postoperative RD rates between phakic and pseudophakic eyes, this information might be clinically significant. Visualization of the far periphery is more difficult in phakic eyes, possibly allowing small anterior retinal tears to go un-noticed. In addition, in patients undergoing phacovitrectomy, the surgeon is less likely to perform aggressive scleral indentation in an eye that had just undergone an anterior chamber procedure. Furthermore, the pupil often constricted slightly as the surgery is lengthened, again inhibiting good visualization of the retinal periphery.

In our study, we saw more post-operative RD in the PCLR group. While this was not statistically significant, one cannot rule out that some post-operative complications might stem from the adjunct treatment itself. Laser retinopexy is associated with increased inflammation and retinal vasculature permeability [16], and can lead to ERM formation [17]. Such intense equatorial fibrosis can lead to retinal tear formation and subsequent RD. We did not, however, saw any clinical evidence to support such clinical findings. The induction of 
PVD during surgery can also cause iatrogenic retinal breaks [14]. Our medical records do not mention specifically whether a PVD was present before surgery or was induced during the operation. Therefore, we could not account for this risk factor in our analysis. But it should be emphasized that induction of PVD was routinely performed in cases where the posterior hyaloid was still attached to the retina during the operation as part of our routine practice.

The risk for developing post-operative $\mathrm{RD}$ is higher in the early post-operative period $[8,11,12]$. Likewise, most RD cases in our cohort occurred within 90 days of the operation $(83.3 \%, 5 / 6)$. This calls for increased vigilance during the early post-operative period, and for more frequent follow-up visits.

Recommendations for PCLR in specific patients are beyond the scope of our study. Nevertheless, we believe that certain patients such as those with multiple peripheral breaks or lattice areas and high myopia should be considered for PCLR.

Our study has several limitations, mainly its retrospective design. Routine use of PCLR was introduced to our department at the beginning of 2008. However, there were still some cases where PCLR was not performed due to surgeon's discretion, possibly introducing a selection bias. The PCLR group had their surgeries done at a later date, with the benefit of better surgical instruments and methods. However, this should have created a bias towards better results in the PCLR group, which we did not notice. Some of the surgeons did not routinely use PCLR, using it only in patients who they thought had increased risk for $\mathrm{RD}$, thereby introducing selection bias.

\section{CONCLUSION}

In conclusion, PCLR did not reduce the occurrence of postoperative RD in our cohort. PCLR's role in small gauge vitrectomy surgeries is questionable. Our study reinforces the need for meticulous intraoperative inspection for iatrogenic retinal breaks, as well as an intentional examination of the retina periphery during early post-operative follow-up. Further prospective randomly designed studies are warranted in order to fully assess the benefits of PCLR.

\section{LIST OF ABBREVIATIONS}

$$
\begin{array}{ll}
\text { PCLR } & =\text { Prophylactic Circumferential Laser Retinopexy } \\
\text { RD } & =\text { Retinal Detachment } \\
\text { MH } & =\text { Macular Hole } \\
\text { ERM } & =\text { Epiretinal Membrane } \\
\text { PPV } & =\text { Pars Plana Vitrectomy }
\end{array}
$$

\section{ETHICS APPROVAL AND CONSENT TO PARTI- CIPATE}

The study was approved by the ethics committee at the Tel Aviv Medical Center, Tel Aviv, Israel, under approval No. 0204-15.

\section{HUMAN AND ANIMAL RIGHTS}

No animals were used in this research. All human research procedures followed were in accordance with the ethical standards of the committee responsible for human experimentation (institutional and national), and with the
Helsinki Declaration of 1975, as revised in 2013.

\section{CONSENT FOR PUBLICATION}

An informed written consent was obtained from all the patients when they were enrolled.

\section{AVAILABILITY OF DATA AND MATERIALS}

Not applicable.

\section{FUNDING}

None.

\section{CONFLICT OF INTEREST}

None of the authors has any conflict of interest regarding the topics discussed in this paper.

\section{ACKNOWLEDGEMENTS}

Declared none.

\section{REFERENCES}

[1] Guillaubey A, Malvitte L, Lafontaine PO, et al. Incidence of retinal detachment after macular surgery: A retrospective study of 634 cases. Br J Ophthalmol 2007; 91(10): 1327-30.

[http://dx.doi.org/10.1136/bjo.2007.115162] [PMID: 17522152]

[2] Banker AS, Freeman WR, Kim JW, Munguia D, Azen SP. Vitrectomy for Macular Hole Study Group. Vision-threatening complications of surgery for full-thickness macular holes. Ophthalmology 1997; 104(9): 1442-52.

[http://dx.doi.org/10.1016/S0161-6420(97)30118-3] [PMID: 9307639]

[3] Michels RG. Vitrectomy for macular pucker. Ophthalmology 1984; 91(11): 1384-8

[http://dx.doi.org/10.1016/S0161-6420(84)34136-7] [PMID: 6514308]

[4] Jackson TL, Donachie PHJ, Sparrow JM, Johnston RL. United Kingdom National Ophthalmology Database study of vitreoretinal surgery: Report 2, macular hole. Ophthalmology 2013; 120(3): 629-34.

[http://dx.doi.org/10.1016/j.ophtha.2012.09.003] [PMID: 23211634]

[5] Jackson TL, Donachie PHJ, Williamson TH, Sparrow JM, Johnston RL. The royal college of ophthalmologists' national ophthalmology database study of vitreoretinal surgery: Report 4, epiretinal membrane. Retina 2015; 35(8): 1615-21.

[http://dx.doi.org/10.1097/IAE.0000000000000523] [PMID: 25830695]

[6] Tan HS, Lesnik Oberstein SY, Mura M, de Smet MD. Enhanced internal search for iatrogenic retinal breaks in 20-gauge macular surgery. Br J Ophthalmol 2010; 94(11): 1490-2.

[http://dx.doi.org/10.1136/bjo.2009.172791] [PMID: 20494906]

[7] Chang TS, McGill E, Hay DA, et al. Prophylactic scleral buckle for prevention of retinal detachment following vitrectomy for macular hole. Br J Ophthalmol 1999; 83(8): 944-8.

[http://dx.doi.org/10.1136/bjo.83.8.944] [PMID: 10413699]

[8] Koh HJ, Cheng L, Kosobucki B, Freeman WR. Prophylactic intraoperative 360 degrees laser retinopexy for prevention of retinal detachment. Retina 2007; 27(6): 744-9.

[http://dx.doi.org/10.1097/IAE.0b013e318030ebd7]

[PMID: 17621184]

[9] Chalam KV, Murthy RK, Gupta SK, Khetpal V. Prophylactic circumferential intraoperative laser retinopexy decreases the risk of retinal detachment after macular hole surgery. Eur J Ophthalmol 2012; 22(5): 799-802.

[http://dx.doi.org/10.5301/ejo.5000115] [PMID: 22344467]

[10] Iwase T, Jo YJ, Oveson BC. Effect of prophylactic $360^{\circ}$ laser treatment for prevention of retinal detachment after phacovitrectomy: (Prophylactic $360^{\circ}$ laser treatment for prevention of retinal detachment). BMC Ophthalmol 2013; 13: 77. [http://dx.doi.org/10.1186/1471-2415-13-77] [PMID: 24325585]

[11] Kim C-S, Kim K-N, Kim W-J, Kim J-Y. Intraoperative endolaser retinopexy around the sclerotomy site for prevention of retinal detachment after pars plana vitrectomy. Retina 2011; 31(9): 1772-6. [http://dx.doi.org/10.1097/IAE.0b013e31820b6129]
[PMID: 
21478805]

[12] Tosi GM, Esposti $\mathrm{P}$, Romeo $\mathrm{N}$, et al. The incidence of rhegmatogenous retinal complications in macular surgery after prophylactic preoperative laser retinopexy: A retrospective study. Medicine (Baltimore) 2016; 95(14)e3283

[http://dx.doi.org/10.1097/MD.0000000000003283] [PMID: 27057893]

[13] Tarantola RM, Tsui JY, Graff JM, et al. Intraoperative sclerotomyrelated retinal breaks during 23-gauge pars plana vitrectomy. Retina 2013; 33(1): 136-42.

[http://dx.doi.org/10.1097/IAE.0b013e31825e1d62]

[PMID: 22791174]

[14] Chung SE, Kim KH, Kang SW. Retinal breaks associated with the induction of posterior vitreous detachment. Am J Ophthalmol 2009;
147(6): 1012-6

[http://dx.doi.org/10.1016/j.ajo.2009.01.013] [PMID: 19327743]

[15] Neffendorf JE, Gupta B, Williamson TH. Intraoperative complications of patients undergoing small-gauge and 20-gauge vitrectomy: A database study of 4,274 procedures. Eur J Ophthalmol 2017; 27(2): 226-30.

[http://dx.doi.org/10.5301/ejo.5000856] [PMID: 27646328]

[16] Nonaka A, Kiryu J, Tsujikawa A, et al. Inflammatory response after scatter laser photocoagulation in nonphotocoagulated retina. Invest Ophthalmol Vis Sci 2002; 43(4): 1204-9.

[PMID: 11923267]

[17] Blackorby BL, Jeroudi AM, Blinder KJ, Shah GK. Epiretinal membrane formation after treatment of retinal breaks: Cryoretinopexy versus laser retinopexy. Ophthalmol Retina 2019; 3(12): 1087-90. [http://dx.doi.org/10.1016/j.oret.2019.06.015] [PMID: 31446030]

\section{C) 2020 Ohana et al.}

This is an open access article distributed under the terms of the Creative Commons Attribution 4.0 International Public License (CC-BY 4.0), a copy of which is available at: (https://creativecommons.org/licenses/by/4.0/legalcode). This license permits unrestricted use, distribution, and reproduction in any medium, provided the original author and source are credited. 